\title{
Patent foramen ovale and left atrial appendage flow velocity predict atrial fibrillation recurrence post cryoballoon ablation
}

\author{
Grzegorz Kiełbasa', Agnieszka Bednarek², Adam Bednarski', Agnieszka Olszanecka', Tomasz Sondej², \\ Aleksander Kusiak², Wiktoria Wojciechowska', Marek Rajzer', Marek Jastrzębski' \\ $11^{\text {st }}$ Department of Cardiology, Interventional Electrocardiology and Hypertension, Jagiellonian University Medical College, Kraków, Poland \\ ${ }^{2}$ Department of Cardiology, Interventional Electrocardiology and Hypertension, University Hospital in Krakow, Kraków, Poland
}

\section{Editorial}

by Spence et al.,

see p. 725

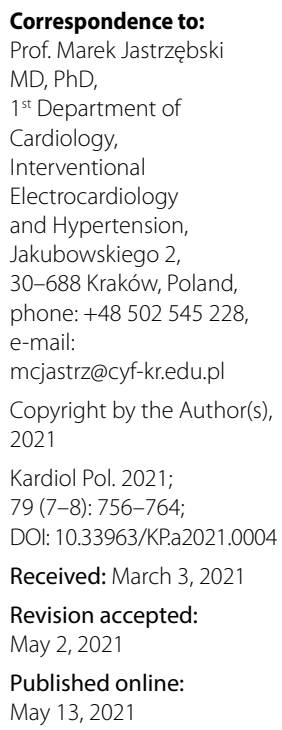

Correspondence to: Prof. Marek Jastrzębski MD, PhD,

$1^{\text {st }}$ Department of Cardiology,

Interventional

Electrocardiology and Hypertension, Jakubowskiego 2 , 30-688 Kraków, Poland, phone: +48 502545228 , e-mail:

mcjastrz@cyf-kr.edu.pl Copyright by the Author(s), 2021

Kardiol Pol. 2021;

79 (7-8): 756-764;

DOl: 10.33963/KP.a2021.0004

Received: March 3, 2021

Revision accepted:

May 2, 2021

Published online:

May 13, 2021

\section{A B S T R A T}

Background: Transesophageal echocardiography (TEE) allows detailed characterization of atrial fibrillation (AF) substrate and could be valuable for predicting pulmonary vein isolation (PVI) procedure outcomes.

Aims: We aimed at assessing the value of TEE-derived left atrial (LA) and LA appendage (LAA) features as prognostic markers for AF recurrence after cryoballoon-based ablation.

Methods: Patients were enrolled using a prospective database of consecutive PVI procedures performed over a 7-year period. The following TEE-derived parameters were investigated: LAA emptying flow velocity (LAA-FV), the presence of patent foramen ovale (PFO), LA spontaneous echo contrast, and mitral regurgitation. Diagnosis of AF recurrence was based on scheduled and symptoms triggered ECG monitoring. The Cox's regression model and Kaplan-Meier survival curves were applied for statistical analysis.

Results: A total of 417 consecutive patients who underwent their first PVI using cryoballoon were analyzed (mean age: 59 years). AF recurrence was noted in $25.7 \%$ of patients (median follow-up of 24 months). Four TEE-derived variables had predictive values for AF recurrence: $L A A-F V<45 \mathrm{~cm} / \mathrm{s}$, presence of PFO at resting state, LA spontaneous echo contrast, and mitral regurgitation. In the multivariable model, apart from the transthoracic echocardiography-derived LA size, two TEE-derived features (LAA-FV $<45 \mathrm{~cm} / \mathrm{s}$ and the presence of PFO) remained as independent predictors.

Conclusions: This study proposed a novel TEE-derived AF recurrence risk factor - the presence of PFO - and confirmed the prognostic value of LAA flow velocity in patients undergoing cryoballoon-based AF ablation. These risk factors could be useful in the global assessment of AF recurrence risk and potentially helpful in planning the ablation strategy.

Key words: atrial fibrillation recurrence, cryoballoon ablation, patent foramen ovale, left atrial appendage flow velocity

Kardiol Pol 2021; 79, 7-8: 756-764

\section{INTRODUCTION}

Atrial fibrillation (AF) ablation is the most commonly performed interventional procedure for arrhythmia treatment. Despite significant progress and the development of multiple AF ablation techniques, the long-term AF recurrence rate remains high. The assessment of the risk factors for $\mathrm{AF}$ recurrence is important for both patient selection/counseling and understanding the reasons behind the failure of this treatment modality.
The most recognized and validated risk factors for AF recurrence include the type of AF (paroxysmal vs persistent), left atrium (LA) enlargement, impaired systolic function of the left ventricle, and comorbidities such as obesity, hypertension, diabetes mellitus, or obstructive sleep apnea [1-3]. Transesophageal echocardiography (TEE) can potentially provide a more accurate risk assessment as it allows the characterization of the AF substrate, i.e., the aspects of morphology and function of the LA and LA 
WHAT'S NEW?

This is the first study that reported the prognostic value of patent foramen ovale for predicting atrial fibrillation recurrence after ablation. The presence of patent foramen ovale doubles the risk of unfavorable long-term results of pulmonary vein isolation with cryoballoon ablation. In the largest study to date, we also showed the relationship between the left atrial appendage flow velocity and atrial fibrillation recurrence in patients after cryoballoon ablation procedure. Consequently, this study corroborated the data that indicated that left atrial appendage flow velocity is an independent and important predictor of atrial fibrillation recurrence.

appendage (LAA), which are unobtainable in the transthoracic echocardiography (TTE). Several studies have shown that features obtained from the TEE have independent prognostic values [4-11].

In this study, we aimed to investigate the value of TEE-derived parameters, concentrating on LA and LAA size and function as prognostic markers of long-term outcomes after a cryoballoon-based pulmonary vein isolation (PVI) procedure.

\section{METHODS}

\section{Study population}

This retrospective analysis was based on our prospectively maintained registry of all consecutive AF ablation procedures performed in patients with symptomatic AF over a 10-year period (June 2009-September 2019). We screened 617 patients; subjects with persistent AF $(\mathrm{n}=122)$, paroxysmal AF with arrhythmia episode during TEE examination (7), incomplete data $(n=20)$, ablation performed with radiofrequency current technique $(n=8)$ or with cryoballoon type 1 ( $n=26)$, or re-do procedures ( $n=17$ ) were excluded. Consequently, the studied group included 417 patients with paroxysmal AF who underwent their first PVI ablation procedure using cryoballoon type
2 (Arctic Front Advance, Medtronic Inc., Minneapolis, MN, USA). The study flowchart was presented in Figure 1. The study was approved by the institutional ethical committee.

\section{Echocardiographic examination}

Each patient underwent a TTE study to obtain the standard dimensions and parameters. In the next step, a TEE examination was performed using a Vivid 9 (GE Healthcare, Horten, Norway) device with a 4D TEE probe on the day the AF ablation was scheduled to assess LA and LAA. To exclude any thrombus, LAA was visualized in the mid-esophageal aortic valve short-axis view $\left(30^{\circ}-60^{\circ}\right)$. Then, the probe was flexed anteriorly and rotated from an angle of $0^{\circ}$ to $180^{\circ}$; placing the pulsed-wave sample volume in the proximal one-third segment of the LAA, and the flow velocities were recorded. The mean value of the LAA late diastolic emptying flow velocity from three consecutive cardiac cycles during regular sinus rhythm was recorded for further analysis. TEE was also used for the detection of LA spontaneous echo contrast. Possible leakage through interatrial septum was tested with Doppler echocardiography, both at rest and after the injection of intravenous contrast (9 $\mathrm{ml}$ of $5 \%$ dextrose solution shaken with $1 \mathrm{ml}$ of ambient air) and Valsalva maneuver. Figure 2 presents examples of investigated features in TEE examination.

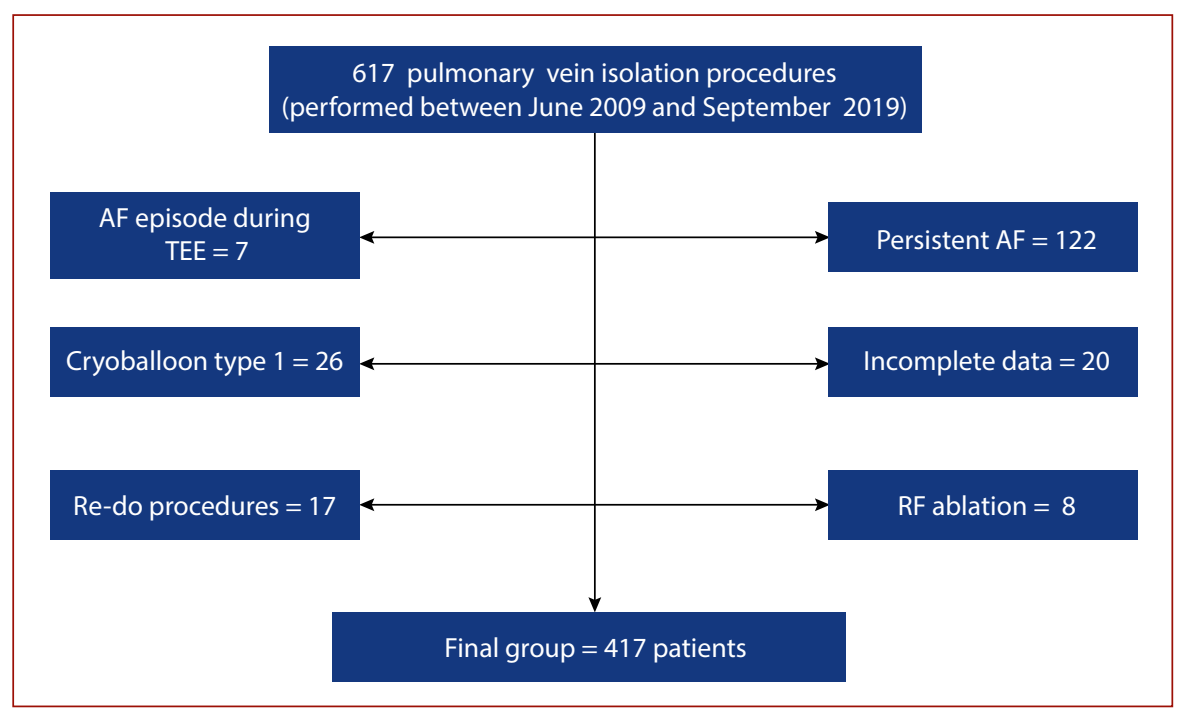

Figure 1. Study flow-chart illustrating reasons for exclusion from the study.

Abbreviations: $\mathrm{AF}$, atrial fibrillation; $\mathrm{RF}$, radiofrequency ablation; $\mathrm{TEE}$, transesophageal echocardiography 


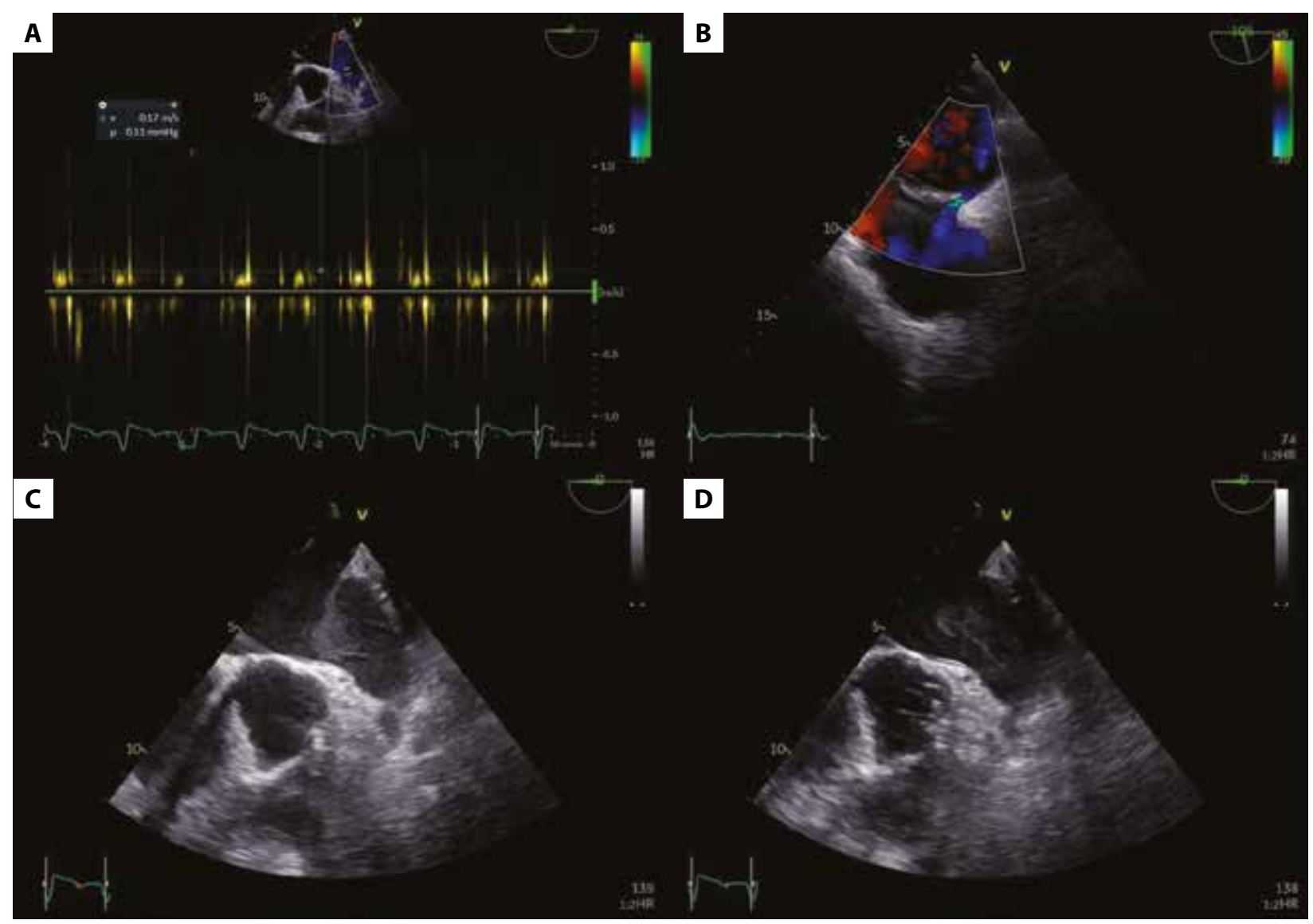

Figure 2. Transesophageal echocardiography. A. Left atrial appendage flow velocity measurement. B. Visualization of blood flow through the patent foramen ovale. C, D. Left atrial spontaneous echo contrast

\section{Ablation procedure}

All PVI procedures were performed under conscious sedation using a $28 \mathrm{~mm}$ cryoballoon. No prior pulmonary vein anatomy assessment was performed; if considered necessary, pulmonary vein anatomy was assessed with contrast/angiography during the procedure. A His bundle catheter and coronary sinus catheter were used to facilitate the transseptal puncture and also for phrenic nerve pacing and electrophysiological assessment of PV isolation. Transseptal puncture was used as an access method to the left atrium in all included patients, including patients with patent foramen ovale (PFO). No attempts to cross PFO with guidewire or ablation catheter were made. Such strategy was based on the considerations that PFO is situated more superior and anterior in comparison to the ideal access site made with the use of transseptal puncture. As a result, the transPFO access may limit the access to pulmonary veins and hinder the successful creation of permanent lesions around PVs' ostia. Our approach/strategy of cryoballoon-based PVI was described in detail elsewhere $[12,13]$ and generally followed the expert consensus recommendations [14]. The procedural endpoint was the electrical isolation of all PVs.

\section{Follow-up and the study endpoint}

The analyzed endpoint of this study was freedom from $\mathrm{AF}$ recurrence. AF recurrence was defined as the first episode of AF lasting $>30$ seconds diagnosed after the blanking period of 3 months. Atrial fibrillation had to be documented by ECG, Holter monitoring, or intracardiac electrogram from the implanted device. Holter monitoring (24-72 hours long) was scheduled after 3 and 6-9 months post-ablation and then advised once a year. Moreover, all patients were advised to obtain an ECG each time they experienced palpitations. Data regarding the primary endpoint were obtained prospectively during the scheduled follow-up visits. However, additionally, all patients received a telephone call at the time of conducting this study to ensure the accuracy of the database concerning the study endpoint. Antiarrhythmic drugs were discontinued either immediately post-ablation or at the latest after the 3-month blanking period.

\section{Statistical analysis}

Continuous variables are presented as means and standard deviations or medians with interquartile range (IQR) while categorical variables are presented as numbers and percentages. In order to confirm the normal distribution, the Shapiro-Wilk test was used. Comparisons between groups were performed using Student's t-test for independent variables (continuous variables) and the chi-square test (proportions). Freedom from AF (time to first AF re-occurrence) after the ablation was assessed with the use of the 
Table 1. Baseline characteristics of the study population $(n=417)$

\begin{tabular}{|c|c|c|c|c|}
\hline Parameters & $\begin{array}{l}\text { Whole group } \\
\qquad(n=417)\end{array}$ & $\begin{array}{l}\text { No AF recurrence } \\
\qquad(n=310)\end{array}$ & $\begin{array}{l}\text { AF recurrence } \\
(n=107)\end{array}$ & P-value \\
\hline Age, year, mean (SD) & $59(11)$ & $58(11)$ & $61(9)$ & 0.03 \\
\hline Male gender, n (\%) & $253(60.7)$ & $194(62.6)$ & $59(55.4)$ & 0.49 \\
\hline $\mathrm{BMI}, \mathrm{kg} / \mathrm{m}^{2}$, median (IQR) & $28.8(25.9-31.5)$ & $28.4(25.7-31.5)$ & $29.5(27-32)$ & 0.03 \\
\hline AF history, months, median (IQR) & $48(24-72)$ & $36(24-77)$ & $60(24-72)$ & 0.28 \\
\hline Use of AA drugs $>2, n(\%)$ & $41(11.3)$ & $30(9.7)$ & $17(15.9)$ & 0.08 \\
\hline Current/past smoker, n (\%) & $124(29.7) / 25$ (6.0) & $90(29.0) / 20(6.5)$ & 34 (31.8) / 5 (4.7) & 0.72 \\
\hline $\mathrm{CHA}_{2} \mathrm{DS}_{2}$-VASc score ${ }^{\mathrm{a}}$, median (IQR) & $2(1-3)$ & $2(1-3)$ & $2(1-3)$ & 0.01 \\
\hline \multicolumn{5}{|l|}{ Comorbidities } \\
\hline Hypertension, n (\%) & $277(66.4)$ & $198(63.9)$ & $79(73.8)$ & 0.06 \\
\hline Diabetes mellitus, n (\%) & $55(13.2)$ & $35(11.3)$ & $20(18.7)$ & 0.051 \\
\hline Chronic coronary syndrome, $\mathrm{n}(\%)$ & $34(8.2)$ & $26(8.4)$ & $8(7.5)$ & 0.77 \\
\hline Heart failure, n (\%) & $13(3.1)$ & $8(2.6)$ & $5(4.7)$ & 0.28 \\
\hline Structural heart disease ${ }^{\mathrm{a}}, \mathrm{n}(\%)$ & $30(7.2)$ & $21(6.8)$ & $9(8.4)$ & 0.57 \\
\hline Stroke/TIA, n (\%) & $19(4.6)$ & $11(3.6)$ & $8(7.48)$ & 0.09 \\
\hline eGFR $<60 \mathrm{l} / \mathrm{min} / 1.73 \mathrm{~m}^{2}, \mathrm{n}(\%)$ & $49(11.8)$ & $35(11.3)$ & $14(13.1)$ & 0.62 \\
\hline
\end{tabular}

aDefined as cardiomyopathy or artificial valve or severe valvular disease or left ventricular ejection fraction $<50$.

Abbreviations: $\mathrm{AA}$, antiarrhythmic; $\mathrm{AF}$, atrial fibrillation; $\mathrm{BMI}$, body mass index; eGFR, estimated glomerular filtration rate; $\mathrm{SD}$, standard deviation; $\mathrm{TIA}$, transient ischemic attack

Table 2. Echocardiographic characteristics of the study population

\begin{tabular}{|c|c|c|c|c|}
\hline Parameters & $\begin{array}{l}\text { Whole group } \\
\qquad(n=417)\end{array}$ & $\begin{array}{l}\text { No AF recurrence } \\
(n=310)\end{array}$ & $\begin{array}{l}\text { AF recurrence } \\
(n=107)\end{array}$ & P-value \\
\hline \multicolumn{5}{|l|}{ TTE } \\
\hline LA dimension, PLAX, mm, median (IQR) & $42(38-45)$ & $41(38-44)$ & $43(40-47)$ & $<0.001$ \\
\hline LV ejection fraction, median (IQR) & $60(60-65)$ & $60(60-65)$ & $60(55-65)$ & 0.11 \\
\hline LVEDD, mm, median (IQR) & $49(46-53)$ & $49(45-53)$ & $50(46-55)$ & 0.35 \\
\hline \multicolumn{5}{|l|}{ TEE } \\
\hline LAA-FV, cm/s, median (IQR) & $57(47-70)$ & $59(49-72)$ & $53(42-67)$ & 0.02 \\
\hline LAA-FV $<45 \mathrm{~cm} / \mathrm{s}, \mathrm{n}(\%)$ & $89(21.3)$ & $59(19.0)$ & $30(28.0)$ & 0.04 \\
\hline LA spontaneous contrast, $\mathrm{n}(\%)$ & $55(13.2)$ & $34(11.0)$ & $21(19.6)$ & 0.02 \\
\hline Patent FO (at rest), n (\%) & $34(8.2)$ & $20(6.5)$ & $14(13.1)$ & 0.03 \\
\hline Patent FO (Valsalva) ${ }^{\mathrm{a}}$, n (\%) & $19(4.6)$ & $16(5.2)$ & $3(2.8)$ & 0.31 \\
\hline Mitral regurgitation & & & & 0.23 \\
\hline Absent or trace, $\mathrm{n}(\%)$ & $205(49.2)$ & $161(51.9)$ & $44(41.1)$ & \\
\hline Mild, n (\%) & $160(38.4)$ & $115(37.1)$ & $45(42.1)$ & \\
\hline Medium, n (\%) & $51(12.2)$ & $33(10.7)$ & $18(16.8)$ & \\
\hline Severe, n (\%) & $1(0.2)$ & $1(0.3)$ & 0 & \\
\hline
\end{tabular}

aTransition of contrast micro-bubbles through foramen ovale during Valsalva maneuver.

Abbreviations: AF, atrial fibrillation; FO, foramen ovale; LA, left atrial; LAA-FV, left atrium appendage late diastolic emptying flow velocity; LV, left ventricle; LVEDD, left ventricular end-diastolic diameter; PLAX, parasternal long-axis view; TEE, transesophageal echocardiography; TTE, transthoracic echocardiography

Kaplan-Meier curve. Survival distribution between groups was compared using the log-rank test. The cut-off point of LAA flow velocity value was calculated based on ROC curve using post hoc data. Univariable and multivariable survival analyses were performed to describe the effect of predictors of AF-free survival. All variables believed to be clinically important were pre-specified and entered into the multivariable Cox regression model. Subsequently, the Wald statistic values were used for stepwise elimination of insignificant variables. The results of Cox models were presented as hazard ratios (HRs) along with tests of significance and $95 \%$ confidence intervals $(\mathrm{Cl})$. There were no significant violations of the proportionality assumption that underlies the Cox proportional hazard method. Statistical analysis was performed in Statistica Software version 13.1 (TIBCO
Software Inc. Palo Alto, CA, USA). A $P<0.05$ was considered statistically significant.

\section{RESULTS}

\section{Patient characteristic and follow-up}

The studied group consisted of 417 patients aged 59 [11] years (mean [SD]), most of them with at least one comorbidity $(71.7 \%)$ and unsuccessful treatment with at least one $(38.9 \%)$, two $(27.1 \%)$, or more $(11.4 \%)$ antiarrhythmic drugs. Detailed baseline clinical and echocardiographic characteristics of this group are presented in Table 1 and Table 2, respectively.

The 7-year observation period resulted in a median follow-up time of 24 months (IQR, 15.5-45.6) - 11056 pa- 
Table 3. Predictors of atrial fibrillation recurrence in univariable and multivariable survival analysis

\begin{tabular}{|c|c|c|c|c|}
\hline Predictor & $\begin{array}{c}\text { Univariable analysis } \\
\text { HR }(95 \% \mathrm{Cl})\end{array}$ & P-value & $\begin{array}{l}\text { Multivariable analysis } \\
\text { HR }(95 \% \mathrm{CI})\end{array}$ & P-value \\
\hline Male sex & $0.77(0.52 ; 1.12)$ & 0.17 & - & \\
\hline BMI per $1 \mathrm{~kg} / \mathrm{m}^{2}$ & $1.05(1.01 ; 1.09)$ & 0.03 & - & \\
\hline Age per 10 years & $1.31(1.08 ; 1.6)$ & 0.006 & - & \\
\hline Hypertension & $1.58(1.02 ; 2.43)$ & 0.04 & - & \\
\hline Diabetes mellitus & $1.75(1.08 ; 2.85)$ & 0.02 & - & \\
\hline Stroke/TIA in history & $1.84(0.89 ; 3.77)$ & 0.1 & - & \\
\hline Chronic coronary syndrome & $0.95(0.46 ; 1.95)$ & 0.89 & - & \\
\hline Structural heart disease & $1.38(0.7 ; 2.74)$ & 0.35 & - & \\
\hline Heart Failure NYHA class $\geq I I$ & $1.84(0.75 ; 4.52)$ & 0.18 & - & \\
\hline Current smoker & $1.12(0.74 ; 1.68)$ & 0.6 & - & \\
\hline $\mathrm{eGFR}<60 \mathrm{l} / \mathrm{min} / 1.73 \mathrm{~m}^{2}$ & $1.24(0.71 ; 2.17)$ & 0.46 & - & \\
\hline LA dimension $>40 \mathrm{~mm}$ & $1.97(1.29 ; 3.01)$ & 0.002 & $1.88(1.23 ; 2.87)$ & 0.004 \\
\hline LV ejection fraction per $10 \%$ & $0.8(0.63 ; 1.02)$ & 0.07 & - & \\
\hline LVEDD per $10 \mathrm{~mm}$ & $1.19(0.84 ; 1.69)$ & 0.32 & - & \\
\hline LA spontaneous contrast & $1.69(1.05 ; 2.73)$ & 0.03 & - & \\
\hline LAA-FV $<45 \mathrm{~cm} / \mathrm{s}$ & $1.73(1.14 ; 2.65)$ & 0.01 & $1.63(1.06 ; 2.49)$ & 0.02 \\
\hline Patent FO (at rest) & $1.88(1.07 ; 3.31)$ & 0.03 & - & \\
\hline Patent FO (Valsalva) ${ }^{\mathrm{a}}$ & $2.02(0.64 ; 6.36)$ & 0.23 & $1.79(1.02 ; 3.15)$ & 0.04 \\
\hline Any mitral regurgitation & $1.55(1.05 ; 2.28)$ & 0.03 & - & \\
\hline
\end{tabular}

Transition of contrast micro-bubbles through foramen ovale during Valsalva maneuver.

Abbreviations: $\mathrm{BMI}$, body mass index; $\mathrm{Cl}$, confidence interval; eGFR, estimated glomerular filtration rate; $\mathrm{FO}$, foramen ovale; HR, hazard ratio; LA, left atrial; LAA-FV, left atrium appendage late diastolic emptying flow velocity; LV, left ventricular; LVEDD, left ventricle end-diastolic diameter; NYHA, New York Heart Association; TIA, transient ischemic attack

tient-months in total. On average, there were 84 hours of Holter ECG monitoring per patient during the observational period. Additionally, 36 patients had data available from the implanted device capable of AF detection (22 implantable loop recorders and 14 pacemakers). AF recurrence after the blanking period was observed in 107 (25.7\%) patients with a median of 225 days (IQR, 115-550) without AF post ablation.

\section{Clinical and echocardiographic predictors of AF recurrence}

We investigated several clinical and echocardiographic features as potential pre-procedural predictors of $A F$ recurrence. Five non-TEE variables in univariable Cox's regression model showed predictive value: the presence of hypertension and diabetes mellitus, BMI, age, and LA enlargement more than $40 \mathrm{~mm}$ - which was the strongest predictor (Table 3). Four TEE-derived variables were predictive of AF recurrence: LAA flow velocity $<45 \mathrm{~cm} / \mathrm{s}$, the presence of PFO at resting state, LA spontaneous echo contrast, and mitral regurgitation of any degree (Table 3 ).

Kaplan-Meier AF-free survival curves concerning the pre-procedural predictors from TEE are presented in Figure 3 . These factors predicted a $16 \%$ to $20 \%$ absolute difference in the study endpoint at the end of the $4^{\text {th }}$ year. Multivariable survival analysis that included all variables from the univariable analysis showed that two of the TEE-derived variables (LAA flow velocity $<45 \mathrm{~cm} / \mathrm{s}$ and presence of PFO) were independent predictors (Table 3 ), with a $P$ of $<0.001$ for the whole model. The cut-off point of LAA flow velocity value was determined by the ROCcurve analysis.
Additionally, the relation of LAA flow velocity to other TEE-derived parameters was assessed by between-group comparison (Table 4); the presence of LA spontaneous echo contrast and mitral regurgitation was strongly related to the LAA flow velocity value.

\section{DISCUSSION}

The major finding of our study is that LAA flow velocity and the presence of PFO were independent predictors of $A F$ recurrence in a sizable and homogeneous cohort of patients with paroxysmal AF who underwent cryoballoon-based PVI. To the best of our knowledge, this is the first study that reported the relation between PFO and the risk of $A F$ recurrence and the largest study to date showing the relation between LAA flow velocity and arrhythmia recurrence in patients after cryoballoon ablation.

\section{Patent foramen ovale}

We found that PFO is an independent risk factor of AF recurrence with a prognostic impact similar to LA size (HR of 1.79 vs 1.88 , respectively). The relation between $\mathrm{PFO}$ and AF recurrence post ablation is a novel finding and is still not fully understood. However, it is tempting to speculate that there is a causative relationship.

One study found that patients with PFO have "atrial latent vulnerability" to develop tachyarrhythmias due to shorter refractoriness and more frequent arrhythmia inducibility during electrophysiological testing [15]. Moreover, we hypothesize that PFO may also act as an AF trigger - mechanically inducing premature atrial depolarizations.

The increased prevalence of PFO among patients with a migraine headache is well-documented [16]. One of the 


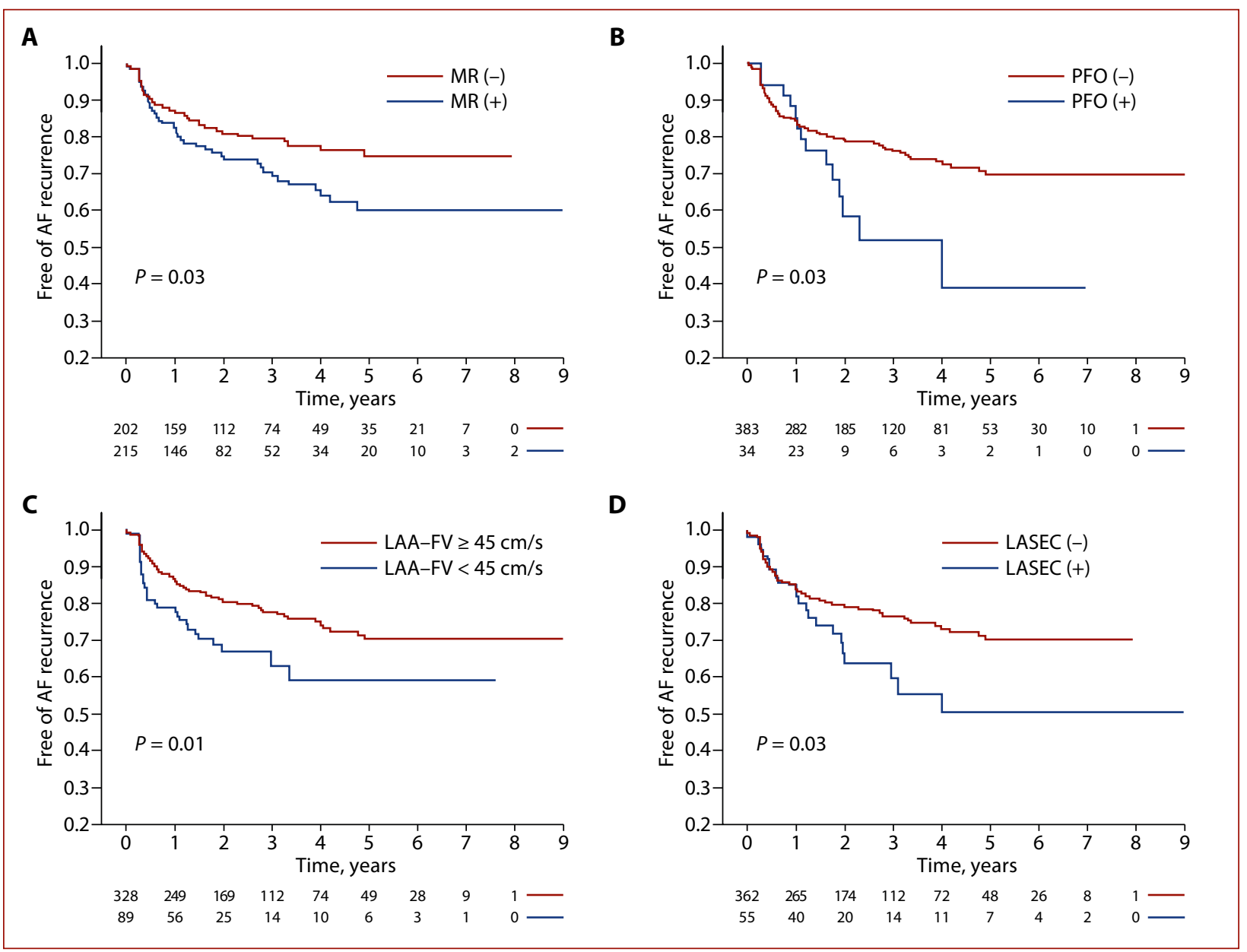

Figure 3. The Kaplan-Meier atrial fibrillation-free survival curves after AF ablation with regard to the pre-procedural transesophageal echocardiography-derived predictors. A. Impact of mitral regurgitation (MR). B. Impact of patent foramen ovale (PFO). C. Impact of left trial appendage flow velocity (LAA-FV). D. Impact of left atrium spontaneous echo contrast (LASEC)

Table 4. Comparison between patients with left atrial flow velocity $<45 \mathrm{~cm} / \mathrm{s}$ and $\geq 45 \mathrm{~cm} / \mathrm{s}$

\begin{tabular}{l|c|c|c} 
& $\begin{array}{c}\text { LAA-FV }<\mathbf{4 5} \mathbf{~ c m} / \mathbf{s} \\
(\mathbf{n}=\mathbf{8 9})\end{array}$ & $\begin{array}{c}\text { LAA-FV } \geq \mathbf{4 5} \mathbf{~ c m} / \mathbf{s} \\
(\mathbf{n}=\mathbf{3 2 8})\end{array}$ & P-value \\
\hline LA spontaneous echo contrast & $37(41.6)$ & $18(5.5)$ & $<0.001$ \\
Patent FO (at rest) & $8(9.0)$ & $26(7.9)$ & 0.75 \\
Patent FO (Valsalva) & $3(3.4)$ & $16(4.9)$ & 0.77 \\
Any mitral regurgitation & $59(66.3)$ & $156(47.6)$ & 0.002 \\
\hline
\end{tabular}

Data are presented as number (percentage) of patients.

aTransition of contrast micro-bubbles through foramen ovale during Valsalva maneuver.

Abbreviations: FO, foramen ovale; LA, left atrial; LAA-FV, left atrium appendage late diastolic emptying flow velocity

postulated mechanisms of this relation evokes the impact of circulating platelet aggregates, serotonin, and other chemicals - which in normal situations are "detoxified" in their first passage through the lungs. A similar mechanism can be operative in the pathogenesis of AF occurrence/recurrence, as influx of venous blood into the LA in patients with PFO may deliver to LA biologically active substances that may predispose to $A F$ initiation or progression of atrial myopathy. Indeed, in a recent work, Daher et al. found an increased prevalence of PFO reaching $57 \%$ in AF patients referred for PVI [17].
It is also possible that PFO is secondary to LA dilatation. Malcoaptation of the edges of the fossa ovale and interatrial shunting may be a consequence of stretching of the interatrial septum - being a part of the process of atrial enlargement [18]. Consequently, PFO could be a marker of structural changes in LA, i.e., a culprit rather than a causative factor. However, this seems a less likely explanation as PFO was found in the current study to be an independent risk factor alongside the LA size. 
Table 5. Left atrial appendage late diastolic emptying flow velocity value as a risk factor for atrial fibrillation recurrence

\begin{tabular}{|c|c|c|c|c|c|c|}
\hline Study & $\mathbf{n}$ & AF type & HR $(\mathrm{CI})$ recurrence & $\mathrm{LAA}-\mathrm{FV}, \mathrm{cm} / \mathrm{s}$ & Ablation method & $\begin{array}{l}\text { Follow-up, } \\
\text { months }\end{array}$ \\
\hline Kiełbasa 2021 & 417 & Parox & $1.63(1.06 ; 2.49)$ & $\geq 45$ & $C B$ & 24 \\
\hline Gerede 2016 [6] & 51 & Parox & $1.13(1.11 ; 1.23)$ & $\geq 30$ & $C B$ & 12 \\
\hline Fukushima 2014 [7] & 105 & Parox & $2.69(1.14 ; 6.32)$ & $\geq 64.9$ & RF & 19 \\
\hline Kanda 2015 [8] & 53 & Persist & $3.24(1.24 ; 8.48)$ & $>28$ & $\mathrm{RF}$ & 12 \\
\hline Ma 2016 [9] & 55 & Persist & $1.23(1.06 ; 1.47)$ & $>8$ & RF & 12 \\
\hline Ma 2016 [9] & 65 & Parox & $1.14(1.02 ; 1.27)$ & $>32$ & RF & 12 \\
\hline He 2018 [10] & 80 & Parox & $1.1(1.01 ; 1.19)$ & $\geq 39.2$ & RF & 12 \\
\hline Kim 2019 [11] & 2352 & Parox and Persist & $2.35(1.97 ; 2.79)$ & $>40$ & $\mathrm{RF}$ & 51 \\
\hline
\end{tabular}

Abbreviations: AF, atrial fibrillation; $C B$, cryoballoon ablation; $C l$, confidence interval; $H R$, hazard ratio; LAA-FV, left atrium appendage late diastolic emptying flow velocity; parox, paroxysmal; persist, persistent; $\mathrm{RF}$, radiofrequency ablation

\section{LAA flow velocity}

LAA flow velocity was validated as an indicator of LA contractile and reservoir function [19]. The relationship between reduced LAA flow velocity and increased risk of AF recurrence after PVI has previously been reported in several studies - summarized in Table 5 [6-11]. However, most of these studies were based on small cohorts and only one study investigated patients who underwent cryoballoon-based ablation [6]. Gerede et al. [6] studied 51 patients who underwent cryoballoon ablation and found that LAA flow velocity was an independent risk factor of AF; our results corroborate this with an analysis based on a group of almost 10 times the size than in the previously studied population. Furthermore, in our study, all patients during TEE were in sinus rhythm, in contrast to some other reports. This seems important, since including patients with persistent AF or patients with AF episodes during TEE and analyzing them together with patients in sinus rhythm results in a serious methodological limitation, as during AF the LAA flow velocity is temporarily decreased.

The LAA flow velocity cut-off point among most studies was similar; any small differences between studies were probably caused by dissimilarities in studied populations and methodology. Our population was relatively healthy, most commonly with hypertension as the only important comorbidity. In the largest LAA flow velocity study to date, a cut-off of $40 \mathrm{~cm} / \mathrm{s}$ was proposed - a value slightly lower than in our study $(45 \mathrm{~cm} / \mathrm{s})$. This could be attributable to the inclusion of patients with persistent $A F$ in that study, as velocity cut-off for persistent AF patients is much lower [9].

\section{Presence of LA spontaneous echo contrast}

LA remodeling related to AF progression manifests in decreased LA contractility development of LA spontaneous echo contrast that can be considered as a final result of LA remodeling, fibrosis, dilation, and decreased hemodynamic function [20]. The presence of LA spontaneous echo contrast as an important independent risk factor of AF recurrence in similar populations was reported by Gerede et al. [6]. However, in our research, this factor was not an independent factor - because it was strongly related to LAA flow velocity (Table 4).

\section{Clinical implications}

AF recurrence is reported to occur in $10 \%-50 \%$ of patients post-cryoballoon ablation. Although several risk factors for AF recurrence were proposed, pre-procedural risk assessment is still far from perfect. A few reports pointed out that other features concerning LA and LAA size and function - like morphological type of LAA, LA strains, and LA pressure, might offer better risk assessment than the standard clinical and TTE-derived measures [4, 21-23].

This study demonstrated that two TEE-derived measures are independent predictors for AF recurrence and potentially could be helpful to distinguish patients with paroxysmal AF who would have a preferable outcome by cryoballoon ablation from those who would not, and could improve the clinical decision-making in these patients. Moreover, it seems justified to speculate that patients with the presence of the above risk markers might benefit from more extensive cryoballoon-based LA ablation (roof and posterior wall lines, LAA appendage isolation, etc.) [24-26] in order to reduce the AF recurrence.

Low LAA flow velocity probably reflects functional atrial remodeling or LAA remodeling related to increased arrhythmogenicity. This seems similar to the observation that the left ventricular ejection fraction predicts the occurrence of ventricular tachyarrhythmias. Since LAA flow velocity remained an independent factor after adjustment for a very potent risk factor - LA size - this suggests that LAA function deterioration may precede LA dilatation and may occur in the early stages of LA remodeling, while the presence of PFO may reflect particular structural atrial remodeling that takes place before global dilatation is observed.

\section{Limitations}

This was a single-center observational study with related potential referral and treatment bias. It is necessary to validate our results with multicenter data analysis. Since arrhythmia recurrences can be asymptomatic and periodic ECG monitoring is far from perfect, undoubtedly some episodes of asymptomatic AF were missed. However, asymptomatic episodes were likely distributed equally in both groups - with and without recognized 
AF limiting the impact of missed AF episodes on the obtained results.

It is possible that we underestimated the true prevalence of PFO. One study showed that TEE is less sensitive in the detection of PFO than gentle attempts to cross the interatrial septum with the use of a steerable catheter during the PVI procedure [17]. However, the prognostic importance of small PFO that does not manifest in TEE during the Valsalva maneuver might be different from the importance of a more "manifest" PFO — as investigated in our population.

The cut-off point of LAA flow velocity was estimated on the basis of post hoc data analysis, potentially leading to the bias of the test validity. However, this type of bias has a higher impact on the test performance in studies with a small sample size or low prevalence of the analyzed effect [26].

\section{CONCLUSIONS}

This study proposed a novel TEE-derived AF recurrence risk factor - the presence of patent foramen ovale - and confirmed the prognostic value of left atrium appendage late diastolic emptying flow velocity during sinus rhythm in patients undergoing cryoballoon-based AF ablation. These risk factors could be useful in the global assessment of AF recurrence risk and potentially helpful in planning the ablation procedure strategy.

\section{Article information}

Conflict of interest: None declared.

Open access: This article is available in open access under Creative Common Attribution-Non-Commercial-No Derivatives 4.0 International (CC BY-NC-ND 4.0) license, allowing to download articles and share them with others as long as they credit the authors and the publisher, but without permission to change them in any way or use them commercially. For commercial use, please contact the journal office at kardiologiapolska@ptkardio.pl.

How to cite: Kiełbasa G, Bednarek A, Bednarski A, et al. Patent foramen ovale and left atrial appendage flow velocity predict atrial fibrillation recurrence post cryoballoon ablation. Kardiol Pol. 2021; 79(7-8): 756-764, doi: 10.33963/KP.a2021.0004.

\section{REFERENCES}

1. Kiełbasa G, Bednarek A, Bednarski A, et al. Predictors of atrial fibrillation recurrence after catheter ablation: data from the German ablation registry. Sci Rep. 2017; 7(1): 16678, doi: 10.1038/s41598-017-16938-6, indexed in Pubmed: 29192223.

2. Liżewska-Springer A, Dąbrowska-Kugacka A, Lewicka E, et al. Echocardiographic predictors of atrial fibrillation recurrence after catheter ablation: a literature review. Cardiol J. 2020; 27(6): 848-856, doi: 10.5603/CJ.a2018.0067, indexed in Pubmed: 29924375.

3. Knight BP, Novak PG, Sangrigoli R, et al.STOP AF PAS Investigators. LongTerm outcomes after ablation for paroxysmal atrial fibrillation using the second-generation cryoballoon: final results from STOP AF post-approval study. JACC Clin Electrophysiol. 2019; 5(3): 306-314, doi: 10.1016/j. jacep.2018.11.006, indexed in Pubmed: 30898232.

4. Kocyigit D, Yalcin MU, Gurses KM, et al. Impact of anatomical features of the left atrial appendage on outcomes after cryoablation for atrial fibrillation. J Cardiovasc Comput Tomogr. 2019; 13(2): 105-112, doi: 10.1016/j. jcct.2019.01.011, indexed in Pubmed: 30639114.
5. Fukushima K, Fukushima N, Kato K, et al. Correlation between left atrial appendage morphology and flow velocity in patients with paroxysmal atrial fibrillation. Eur Heart J Cardiovasc Imaging. 2016; 17(1): 59-66, doi: 10.1093/ehjci/jev117, indexed in Pubmed: 25944049.

6. Gerede DM, Candemir B, Vurgun VK, et al. Prediction of recurrence after cryoballoon ablation therapy in patients with paroxysmal atrial fibrillation. Anatol J Cardiol. 2015 [Epub ahead of print]; 16(7): 482-488, doi: 10.5152/AnatolJCardiol.2015.6309, indexed in Pubmed: 26680545.

7. Fukushima K, Fukushima N, Ejima $\mathrm{K}$, et al. Left atrial appendage flow velocity and time from $P$-wave onset to tissue Doppler-derived $A^{\prime}$ predict atrial fibrillation recurrence after radiofrequency catheter ablation. Echocardiography. 2015;32(7): 1101-1108, doi: 10.1111/echo.12823, indexed in Pubmed: 25362992.

8. Kanda T, Masuda M, Sunaga A, et al. Low left atrial appendage flow velocity predicts recurrence of atrial fibrillation after catheter ablation of persistent atrial fibrillation. J Cardiol. 2015; 66(5):377-381, doi: 10.1016/j. jjcc.2015.04.009, indexed in Pubmed: 25982670.

9. Ma XX, Zhang YL, Hu B, et al. Association between left atrial appendage emptying velocity, N-terminal plasma brain natriuretic peptide levels, and recurrence of atrial fibrillation after catheter ablation. J Interv Card Electrophysiol. 2017; 48(3): 343-350, doi: 10.1007/s10840-016-0216-4, indexed in Pubmed: 27943042

10. He Y, Zhang B, Zhu F, et al. Transesophageal echocardiography measures left atrial appendage volume and function and predicts recurrence of paroxysmal atrial fibrillation after radiofrequency catheter ablation. Echocardiography. 2018;35(7): 985-990, doi: 10.1111/echo.13856, indexed in Pubmed: 29509964.

11. Kim YGi, Choi Jl, Boo KiY, et al. Clinical and echocardiographic risk factors predict late recurrence after radiofrequency catheter ablation of atrial fibrillation. Sci Rep. 2019; 9(1): 6890, doi: 10.1038/s41598-019-43283-7, indexed in Pubmed: 31053744.

12. Kiełbasa G, Jastrzębski M. Cryoballoon pulmonary vein isolation as a standard approach for interventional treatment of atrial fibrillation. A review and a practical guide to an effective and safe procedure. Postepy Kardiol Interwencyjnej. 2020; 16(4): 359-375, doi: 10.5114/aic.2020.101760, indexed in Pubmed: 33598008.

13. Jastrzębski M, Kiełbasa G, Fijorek K, et al. Outcomes of atrial fibrillation ablation program based on single-shot techniques. Postepy Kardiol Interwencyjnej. 2020; 16(4): 466-473, doi: 10.5114/aic.2020.101773, indexed in Pubmed: 33598021.

14. Su W, Aryana A, Passman R, et al. Cryoballoon Best Practices II: Practical guide to procedural monitoring and dosing during atrial fibrillation ablation from the perspective of experienced users. Heart Rhythm. 2018; 15(9): 1348-1355, doi: 10.1016/j.hrthm.2018.04.021, indexed in Pubmed: 29684571.

15. Berthet $K$, Lavergne T, Cohen A, et al. Significant association of atrial vulnerability with atrial septal abnormalities in young patients with ischemic stroke of unknown cause. Stroke. 2000; 31(2): 398-403, doi: 10.1161/01. str.31.2.398, indexed in Pubmed: 10657412

16. Hildick-Smith D, Williams TM. Patent foramen ovale and migraine headache. Interv Cardiol Clin. 2017; 6(4): 539-545, doi: 10.1016/j. iccl.2017.05.006, indexed in Pubmed: 28886844.

17. Daher G, Hassanieh I, Malhotra N, et al. Patent foramen ovale prevalence in atrial fibrillation patients and its clinical significance; A single center experience. Int J Cardiol. 2020; 300: 165-167, doi: 10.1016/j.jjcard.2019.11.088, indexed in Pubmed: 31761403.

18. Fadel BM, Husain A, Bakarman $\mathrm{H}$, et al. Spectral Doppler interrogation of the patent foramen ovale-a window to left heart hemodynamics. Echocardiography. 2015; 32(2): 383-389, doi: 10.1111/echo.12723, indexed in Pubmed: 25130954.

19. Verma A, Marrouche NF, Yamada H, et al. Usefulness of intracardiac Doppler assessment of left atrial function immediately post-pulmonary vein antrum isolation to predict short-term recurrence of atrial fibrillation. Am J Cardiol. 2004; 94(7): 951-954, doi: 10.1016/j.amjcard.2004.06.039, indexed in Pubmed: 15464687.

20. Kim YGi, Choi Jl, Kim MN, et al. Non-vitamin K antagonist oral anticoagulants versus warfarin for the prevention of spontaneous echo-contrast and thrombus in patients with atrial fibrillation or flutter undergoing cardioversion: A trans-esophageal echocardiography study. PLoS One. 
2018; 13(1): e0191648, doi: 10.1371/journal.pone.0191648, indexed in Pubmed: 29360845.

21. Nielsen AB, Skaarup KG, Lassen MC, et al. Usefulness of left atrial speckle tracking echocardiography in predicting recurrence of atrial fibrillation after radiofrequency ablation: a systematic review and meta-analysis. Int J Cardiovasc Imaging. 2020; 36(7): 1293-1309, doi: 10.1007/s10554-02001828-2, indexed in Pubmed: 32248332.

22. Park J, Joung B, Uhm JS, et al. High left atrial pressures are associated with advanced electroanatomical remodeling of left atrium and independent predictors for clinical recurrence of atrial fibrillation after catheter ablation. Heart Rhythm. 2014; 11(6): 953-960, doi: 10.1016/j.hrthm.2014.03.009, indexed in Pubmed: 24607916.

23. Liżewska-Springer A, Dąbrowska-Kugacka A, Lewicka E, et al. Biatrial strain as a new predictive marker of successful pulmonary vein ablation in patients with atrial fibrillation and preserved left ventricular function. Kardiol Pol. 2019; 77(4): 471-474, doi: 10.5603/KP.a2019.0016, indexed in Pubmed: 31025652.
24. Yorgun H, Canpolat U, Okşul M, et al. Long-term outcomes of cryoballoon-based left atrial appendage isolation in addition to pulmonary vein isolation in persistent atrial fibrillation. Europace. 2019;21(11): 1653-1662, doi: 10.1093/europace/euz232, indexed in Pubmed: 31504432.

25. Niazi I, Erickson L, Chaudhari A, et al. Cryoballoon pulmonary vein isolation and roof and posterior wall debulking using navik $3 \mathrm{D}^{\mathrm{TM}}$ : a new technique for atrial fibrillation ablation. J Innov Card Rhythm Manag. 2020; 11(1): 3975-3982, doi: 10.19102/icrm.2020.110105, indexed in Pubmed: 32368367

26. Gul EE, Verma A. Cryoballoon in persistent atrial fibrillation: a standardized or individualized approach? Kardiol Pol. 2020; 78(1): 1-3, doi: 10.33963/KP.15153, indexed in Pubmed: 31976928.

27. Ewald B. Post hoc choice of cut points introduced bias to diagnostic research. J Clin Epidemiol. 2006; 59(8): 798-801, doi: 10.1016/j.jclinepi.2005.11.025, indexed in Pubmed: 16828672. 